\title{
Research on the Coordination Mechanism of Value Cocreation of Innovation Ecosystems: Evidence from a Chinese Artificial Intelligence Enterprise
}

\author{
Yu Chen, ${ }^{1,2}$ Yantai Chen $\mathbb{1}^{2},{ }^{2}$ Yanlin Guo, ${ }^{2}$ and Yanfei $\mathrm{Xu}^{2}$ \\ ${ }^{1}$ School of Business Administration, Shanghai Lixin University of Accounting and Finance, Shanghai 201620, China \\ ${ }^{2}$ School of Management, Zhejiang University of Technology, Hangzhou 310023, China \\ Correspondence should be addressed to Yantai Chen; chenyt@zjut.edu.cn
}

Received 20 July 2020; Revised 26 January 2021; Accepted 15 February 2021; Published 25 February 2021

Academic Editor: Zaoli Yang

Copyright $\odot 2021$ Yu Chen et al. This is an open access article distributed under the Creative Commons Attribution License, which permits unrestricted use, distribution, and reproduction in any medium, provided the original work is properly cited.

\begin{abstract}
This paper models the game process of the value cocreation of enterprises based on evolutionary game theory (EGT). The factors influencing value cocreation are found through mathematical analysis. Taking iFLYTEK as an example, a representative enterprise of artificial intelligence (AI) in China, six factors affecting value cocreation are verified, which are the excess return rate, the distribution coefficient of the excess return rate, coordination costs in the system, the cost-sharing coefficient, imitation costs, and penalties. These six factors have a profound impact on value cocreation in the ecosystem. Through the case study of iFLYTEK, it is concluded that innovation ecosystems can enable small- and medium-sized AI enterprises to grow. In order to build a sound ecosystem, we need to establish a mechanism to select partners, reduce the costs of cooperation, and strengthen the protection of intellectual property. At the beginning of the cooperation, it is necessary to establish a mechanism with clear responsibilities, rights, and interests. The conclusion is of great significance to the development of AI enterprises.
\end{abstract}

\section{Introduction}

The fourth industrial revolution is coming, and $\mathrm{AI}$ is beginning to penetrate all aspects of social production. AI will be the focus of the next industrial revolution in science and technology. Major developed countries are also actively deploying frontier research in AI. AI is a historic opportunity, which plays an important role in alleviating the pressure of population aging, promoting the transformation of the industrial structure, and coping with the challenges of sustainable development ("Artificial intelligence has developed into a national strategy in many countries," ChinaByte, October 21, 2016, http://server.chinabyte.com/432/ 13932932.shtml). Lichtenthaler [1] pointed out that, against the background of this rapid technological progress, machines may have a certain degree of technological innovation ability and participate in the creation of new products, systems, and services. Therefore, the impact of AI on business mainly involves knowledge development and utilization. AI can recognize text information and transform images and voices into understandable information, which enriches the data stream. AI is also regarded as an entity that can imitate human thinking via computers [2]. An AI innovation ecosystem has similar characteristics to a natural ecosystem. In the AI ecosystem, enterprises, universities and scientific research institutions, intermediary institutions, governments, and financial institutions offer their respective advantages and professional expertise and exchange flows of material, energy, and information with the external environment. The innovation aggregation effect of interdependence and interaction in the ecosystem continuously improves the overall innovation ability of the AI ecosystem. The AI innovation ecosystem includes two parts: the innovation population of biological components and the environment of nonbiological components. In the innovation population, the boundaries of producers, consumers, and decomposers are not so clear, and there are often interactions or overlaps. For example, producers include 
enterprises, universities, scientific research institutions, and other organizations that develop and apply AI technology. Consumers include enterprises and institutions that apply AI technology to various industries. The decomposer is the users who purchase relevant AI products and provide feedback information to the producer. Through the cooperation among producers, consumers, and decomposers, the ecosystem can develop continuously and improve its operation efficiency.

Botha [3] addressed the possible future evolution of innovation from a human-only initiative to human-machine coinnovation and then autonomous machine innovation, arriving at a conceptual mind model that outlines the role of innovation regimes and innovation agents. Wauters and Vanhoucke [4] provided a nearest neighbor-based extension for project control forecasting with earned value management and selected an AI method to reduce the training set to predict the real duration of a project. Lichtenthaler [5] discussed the interplay between humans and AI. Using AI at IBM Research for product composition, McCormick is transforming workflow processes for product developers, enabling them to create more innovative flavor products faster and to significantly improve their success rates [6]. This has shed light on how companies can create value through AI and highlights the strategic decisions IBM makes to create value in two dimensions: internal development and external collaborations [7]. Research of AI technology applications in business is just beginning. In the existing literature, the AI research pays more attention to AI technology, policy-making, and comparisons of international development, but rarely from the perspectives of value cocreation and innovation ecosystems. In fact, every AI enterprise experiences the ecological cycle from start-up to growth and needs to work out how to cooperate with other business partners in the ecosystem. In the growth process, AI enterprises have to face the important decision of deciding whether to cooperate or not. These problems regarding how to obtain the most favorable resources in an AI ecosystem, how to build cooperation mechanisms with other business partners, and how to find dynamic cooperation strategies are very important for the healthy development of AI enterprises, but the current research is not deep enough.

This paper analyzes the dynamic game process of platform-leading enterprises and related cooperative enterprises in innovation ecosystems of AI by establishing the replication dynamic equation. Through seeking stable solutions for the duplicated dynamic equation, six main factors affecting value cocreation are found using a calculus derivation method. Then, taking the example of iFLYTEK, a leading AI enterprise in China, the game model and the experience of value cocreation between iFLYTEK and its partners are verified. Finally, a strategy for more AI enterprises is suggested. The main contribution of this paper is to explore coordination mechanisms so as to jointly benefit and promote the development of an innovation ecosystem between platform-leading enterprises and related cooperative enterprises and to discuss the factors affecting the value cocreation of such enterprises. Because the coordination process between the two sides has to go through many repeated games in order to establish a relatively stable cooperative relationship, the learning process also shows evolution from a low level to a high level so as to achieve the maximum benefit under limited rationality. It is appropriate to use EGT to analyze the coordination mechanism of value cocreation. By solving the equilibrium of the replication dynamic equation, it is found that both sides choose to cooperate or not, depending on the location of the saddle point, and the saddle point is determined by six factors, which are the excess return rate, the distribution coefficient of the excess return rate, coordination costs in the system, the cost-sharing coefficient, imitation costs, and penalties. Taking iFLYTEK as an example, China's leading AI enterprise, these six factors are verified as affecting value cocreation in an AI ecosystem. The conclusion enriches the research on the value cocreation of innovation ecosystems.

The rest of the paper is arranged as follows. Section 2 reviews the existing studies. Section 3 establishes the theoretical model. Section 4 discusses the factors that may influence the result of the game. Section 5 analyzes the case of iFLYTEK to discuss the application of game evolution. Section 6 concludes the paper and proposes policy implications and future research.

\section{Literature Review}

2.1. Innovation in the AI Industry. With the rapid development of the AI industry, innovation management at the theoretical level has aroused a heated discussion among scholars around the world [8]. The evolution of the AI industry can be divided into three stages. In the first stage, it focuses on perceptual intelligence represented by the deep convolution neural network, which relies on big data. It has achieved success comparable to human intelligence in visual recognition, speech recognition, and natural language understanding. In the second stage, it focuses on cognitive intelligence, including reasoning, planning, memory, decision-making, and knowledge learning. In the third stage, it focuses on creative intelligence; that is to say, AI is required to have super abilities, like the insight and inspiration of human beings. At present, the development of the AI industry is facing many challenges, such as the economy, security, and supervision. Many countries pay special attention to the policies in this field and successively create national policies regarding AI. All kinds of innovative products, systems, and services are created by AI, thus promoting production transformation and social progress. Compared with traditional technological innovation, AI innovation has significantly improved in terms of industry, technology, and social value [9]. On the one hand, AI gradually brings new products to the market, such as computer vision and voice, semantic recognition, and intelligent robots. On the other hand, it helps to transform and upgrade traditional manufacturing and service industries, such as intelligent manufacturing, intelligent logistics, intelligent transportation, and intelligent education. AI can greatly improve the efficiency of research and development (R\&D). It has a far-reaching impact on innovation performance and global manufacturing output and provides 
customers with personalized services, thus creating greater economic and social value.

To understand the impact of choice complexity and cognitive perceptions on the willingness to delegate a strategic decision to an algorithm, an experiment with 310 participants was conducted and found that, although choice complexity has no effect, participants with low levels of situational awareness are more likely to delegate [10]. Boyd and Holton [11] focused on the economic, political, and historical dynamics of technological innovation and its consequences for employment and economic restructuring and mediated through sovereign and discursive power. Elliott [12] pointed out that, as technological innovation has eliminated many types of jobs over the past few centuries, economies have evolved to create new jobs, which have kept workers well employed. Fujii and Managi [13] also applied a decomposition framework to clarify the determinants of AI technology invention and found that the priority has shifted from biological and knowledge-based models to specific mathematical models and other AI technologies. Hengstler et al. [14] explored how firms systematically foster trust regarding applied AI. Based on empirical analysis using nine case studies in the transportation and medical technology industries, their study illustrates the dichotomous constitution of trust in applied AI.

\subsection{Research on Coordination Mechanisms in Innovation} Ecosystems. In the modern society, the competition among enterprises has changed from individual to platform competitors. The complexity of the economic environment also causes enterprises to form more diversified ecological relationships, which are competitive and symbiotic. Moore [15] has put forward the concept of the innovation ecosystem for the first time and then proposed the concept of a business ecosystem, which is more conducive to value creation. Scholars have also studied innovation ecosystems from various perspectives. For example, Leten et al. [16] have put forward the intellectual property (IP) model of the coordinating mechanisms of an innovation ecosystem. The model analyzed knowledge sharing between partners, which can promote the development of the ecosystem. Policy is very important for the innovation ecosystem, and the behaviors of all participants in the ecosystem should be consistent with the strategic objectives of national policies [17]. Liu and Rong [18] took complex products in the mobile Internet industry ecosystem as an example for exploring the coevolution mechanism of enterprise self-renewal. Kapoor and Furr [19] discussed the solar photovoltaic industry to analyze the complementary capabilities of enterprises and the driving mechanism of complementary assets on technology. Lee et al. [20] illustrated the determinants of ICT innovation with a transnational empirical study, which showed that the cooperation of universities, enterprises, and governments effectively promoted knowledge sharing. Spigel [21] believed that a successful innovation ecosystem is a good supporting environment created by the complementary cooperation of all parties involved in the system. Spigel [21] also indicated that the relationship between the parties is an important guarantee for the competitiveness of the system. She constructed different game models using Bayesian networks and game theory, which were helpful in understanding stakeholders' behaviors in the ecosystem service. Mulazzani et al. [22] found that the number of human agents, management actions, and economic activities might largely affect the value of ecosystem service benefits. Madsen [23] provided a foundation for studying a new framework within the cooperation of the global ecosystem for sustainable development and clarified the types of organizational capabilities, practices, and routines that can strengthen both internal value processes and external networks and ecosystems. Moreover, Masucci et al. [24] investigated how firms can orchestrate outbound open innovation strategically to accelerate technological progress among the firms they collaborate with, thus removing technological bottlenecks in their business ecosystems. Xie and Wang [25] explored the causal recipes of how open innovation ecosystem modes promote product innovation by using both grounded theory and fuzzy-set qualitative comparative analysis (fsQCA).

\subsection{Research on Cooperation Mechanisms in Value} Cocreation. From the perspective of literature development, the research on the coordination mechanism of value cocreation is mainly carried out on three aspects. Firstly, research is mainly focused on the contributions of all parties involved in value creation from a systematic level. For example, Vargo and Lusch [26] placed special emphasis on the impact of institutions in the service ecosystem. Secondly, research is focused on coordination mechanisms, which can help participants obtain more knowledge, information, and public resources from the perspective of participating individuals. Vargo and Lusch [27] proposed that the foundation of value cocreation is determined by a binary relationship, which referred to the service interaction between enterprises and customers. Thirdly, the paper discusses coordination mechanisms including excess returns, the costs incurred for coordination, and the possible risks. It is found that opportunities, competition levels, and demand preferences are the driving factors of reverse innovation. Selective disclosure is indicated as a strategic mechanism for reshaping the cooperative behavior of other participants in the innovation ecosystem. Alexy et al. [28] pointed out that selective disclosure may provide an effective alternative, especially in the case of high uncertainty, high coordination costs, and the unwillingness of partners. Leclercq et al. [29] investigated the impact of two gamification mechanics' cooperation and competition, highlighted the existence of four user profiles, and then assessed their emotional, cognitive, and behavioral engagement with the gamified cocreation platform over time. Niesten and Stefan [30] reviewed literature on paradoxical tensions between value cocreation and capture in interorganizational relationships. Su and $\mathrm{Li}$ [31] pointed out internal mechanisms of knowledge transfer in a knowledge alliance and showed that the number of enterprises in the knowledge alliance, knowledge transfer frequency, and knowledge transfer effects is positively correlated. 
2.4. Application of Evolution Game Theory in Industrial Dynamic Evolution. In recent years, EGT has been widely used in the study of industrial dynamic evolution. Wood et al. [32] used an agent-based model to provide detailed results and demonstrated the importance of natural resources for the outcome of the model. Yang et al. [33] analyzed government-industry-university-research (GIUR) intellectual property cooperation behavior and its influencing factors, including market mechanisms and administrative supervision mechanisms. Hafezalkotob et al. [34] used an integrated Cournot duopoly equilibrium and evolutionary game theory (EGT) approach to model the situation where a wholesale pricing strategy is determined by a manufacturer acting as a leader, while retailers who make order quantity decisions are acting. Mekki et al. [35] studied the vehicular cloud access problem and modeled it as an evolutionary game where the vehicles choose to cooperate or to access the conventional cloud.

In summary, although much research has been carried out on coordination mechanisms in innovation ecosystems and value cocreation, there are still gaps in the existing research. First, because of the regional imbalance in the AI industries in many countries such as China, coordination mechanisms may be different. Therefore, this study explores the coordination mechanisms of innovation ecosystems based on an evolutionary game model. Second, although there are several studies on the contributions of all parties involved in value creation and coordination mechanisms, only few studies have focused on the coordination mechanisms of AI industries from the perspective of game theory. Third, AI is an emerging industry in China, and research on the AI innovation ecosystem has just started. It is of great theoretical and practical value to analyze the composition, cooperation, and evolution of Chinese AI innovation ecosystems in terms of their healthy development. This study incorporates these three perspectives.

\section{Model and Analysis}

Many AI enterprises are faced with the pain of transformation from laboratory achievements to product marketization due to large initial technology investment and market risk. However, there is little research on the business model of AI enterprises, how they create value together with their partners, and how they build an innovation ecosystem to empower themselves. Ma and Ji [36] pointed out the synergetic development of regional ecological communities had an important impact on ecosystem evolution, in which the evolution process was gradual, including four stages: synergetic construction, synergetic expansion, synergetic cooperation, and synergetic innovation. Abiodun and Ivan [37] presented a model of interfirm cooperation driven by cognitive distance, appropriability conditions, and external knowledge and found that a firm chooses to cooperate and selects a partner conditional on the investments in absorptive capacity. Brice et al. [38] highlighted how ecosystem creation was a systemic process driven by coupled feedback loops, which organizations must try to control dynamically. Ron [39] followed the flow of inputs and outputs in the ecosystem to distinguish between upstream components that were handled by the local firm and downstream complements that were bundled by the firm's customers. Therefore, the key point of this paper is to explore the coordination mechanism of value cocreation in the innovation ecosystems of AI enterprises. EGT abandons the assumption that participants are completely rational. It regards the behavior of all participants in an ecosystem as contributing to a dynamic system; that is, the actors always aim at fulfilling their own interests. Moreover, it assumes "bounded rationality" for the behavior of participants in the system and pays more attention to the process of reaching an equilibrium. Therefore, EGT is an appropriate method for analyzing the cocreation mechanism between leading enterprises and cooperative enterprises in an AI ecosystem. Time is an important factor in the changes in the relationship between leading enterprises and related cooperative enterprises. Only through games can the two sides achieve a dynamic balance process. By means of EGT, we can clearly depict the dynamic path of equilibrium between leading enterprises and cooperative enterprises in $\mathrm{AI}$ innovation ecosystems.

3.1. Assumptions. In order to analyze the problem of the cocreation mechanism in innovation ecosystems, this paper makes the following assumptions:

(1) AI enterprise innovation ecosystem includes competitors, operators, technology intermediaries, and research institutions. There is a platform-leading enterprise $A$ and a related cooperative enterprise $B$.

(2) The AI innovation ecosystem includes two parts: biological components and the environment. Among them, biological components include producers, consumers, and decomposers. The producer refers to the enterprise engaged in the R\&D and production of AI technology. The consumer refers to many kinds of application that apply AI technology to specific industries, including enterprises, universities, and scientific research institutes. The decomposer refers to the users that purchase relevant AI products.

(3) In the AI innovation ecosystem, the platform-leading enterprise and other enterprises may have a possible cooperative relationship, but this relationship is not permanent, and it may be broken at any time, or a new cooperative relationship may be generated. The choice of cooperative or noncooperative behavior is real-time. Because of the distribution of interests, they play games with each other and determine the game strategy in their interaction, showing the characteristics of the evolutionary game.

(4) Leading enterprise tries to cooperate with other enterprises in the ecosystem with a certain probability at the initial stage and generates excess returns in the process of cooperation. The change of decision-making in the game process essentially stems from the pursuit of higher interests, so the allocation 
of excess returns is the driving force for the development of the AI ecosystem.

(5) When the platform-leading enterprise $A$ and the related cooperative enterprise $B$ are in a state of cooperation, the two sides create value together, and the innovation ecosystem is developing towards a virtuous path. When one performs noncooperative behavior, it is difficult for both to create value together, and there will be many contradictions, even dissolution [40].

(6) The response of a relevant cooperative enterprise $B$ is crucial to the choice of a platform-leading enterprise $A$. Therefore, the strategic choice of both is a dynamic game process, and the best strategy is cooperation, which can maximize benefits, increase the overall profit of the innovation ecosystem, and minimize the total costs and risk [41]. However, both $A$ and $B$ have limited rational. In decision-making, they only consider the maximization of their own interests and adopt a strategy of noncooperation.

(7) The dynamic game process is a mixed strategy game and the diversification of strategy selection. Under certain probability conditions, the game process is adjusted according to the strategy of the other one [42].

(8) As AI enterprises, their technical threshold is very high. In the early stages, they need to invest a lot of energy and cost in developing technology. Their technical threshold is also their core competitiveness in the commercial economy. However, during cooperation, there is also the problem of "hitchhiking" or "stealing" technology [43]. If such a situation occurs, the cost paid by both parties will be much higher than the benefits from cooperation. If the core technology is imitated, the imitator will obtain a technological imitative income. Therefore, in order to protect independent R\&D participants, both of them need to introduce reward and punishment measures when cooperating. If the core technology is imitated or "stolen," one party will give the other a certain amount of compensation and will be punished accordingly.

\subsection{Evolutionary Game Model}

3.2.1. Variables. There are several variables that have positive values in the game, and they are defined as follows:

$P_{1}$ : leading enterprise $A$ can independently obtain maximum profit, which can be obtained from the respective market when there is no cooperative relationship with $B$.

$P_{2}$ : enterprise $B$ can independently obtain maximum profit, which can be obtained from the respective market when there is no cooperative relationship with $A$.

$\Delta R$ : when both $A$ and $B$ cooperate to create value together, it is beneficial for the whole innovation ecosystem and can create additional benefit $\Delta R$. $\alpha: A$ and $B$ will be assigned the additional benefit, and $\alpha$ is a distribution coefficient which $A$ obtains. $\alpha \in(0,1)$. $1-\alpha: 1-\alpha$ is a distributor coefficient of the additional benefit that $B$ obtains.

$C$ : the transaction costs of the ecosystem, which are the negotiation costs and management costs incurred in order to coordinate the behavior of $A$ and $B$.

$\beta: \beta$ is the cost-sharing coefficient for $A .1-\beta$ is the cost-sharing coefficient for $B . \beta \in(0,1)$.

$M_{1}$ : if the core technology is imitated, then $A$ will pay $M_{1}$ in terms of imitation costs.

$M_{2}$ : if the core technology is imitated, then $B$ will pay $M_{2}$ in terms of imitation costs.

$F$ : if the core technology is imitated or "stolen," one party will give the other a certain amount of compensation and will be punished accordingly. $F$ represents penalties. The introduction of penalties is also to protect the cooperation between the two sides.

3.2.2. Payoff Matrix. In the process of the dynamic game, there are four strategies. Figure 1 shows the payoff matrix of the players in the evolutionary game.

\subsubsection{Evolutionary Stable Strategy Applying a Replication} Dynamic Equation. The parameters of $x$ and $y$ are used to indicate the probabilities of platform-leading enterprise $A$ and cooperative enterprise $B$ choosing different strategies, respectively. $E_{a}$ represents the average expected payoffs of enterprise $A . E_{b}$ represents the average expected payoffs of enterprise $B$. Table 1 shows the specifications of all the parameters.

(1) Replication Dynamic System. According to the framework of EGT, we can observe the payoffs of players under different combinations of strategies. For platform-leading enterprise $A$, in the coordination mechanism of value cocreation, the expected return from cooperation is $E_{a 1}$. The expected return from noncooperation is $E_{a 2}$. The average expected return is $E_{a}$. Then, the corresponding formula is as follows:

$$
\begin{aligned}
E_{a 1}= & y\left(P_{1}+\alpha \Delta R-\beta C\right)+(1-y)\left(P_{1}-\beta C+F\right)=y \alpha \Delta R \\
& +(1-y) F+P_{1}-\beta C, \\
E_{a 2}= & y\left(P_{1}-M_{1}-F\right)+(1-y) P_{1}=P_{1}-y M_{1}-y F, \\
E_{a}= & x E_{a 1}+(1-x) E_{a 2}=x y \alpha \Delta R+(x-y) F+P_{1} \\
& -y(1-x) M_{1}-x \beta C .
\end{aligned}
$$

For enterprise $B$, in the coordination mechanism of value cocreation, the expected return from cooperation is $E_{b 1}$. The expected return from noncooperation is $E_{b 2}$. The average expected return is $E_{b}$. Then, the corresponding formula is as follows: 


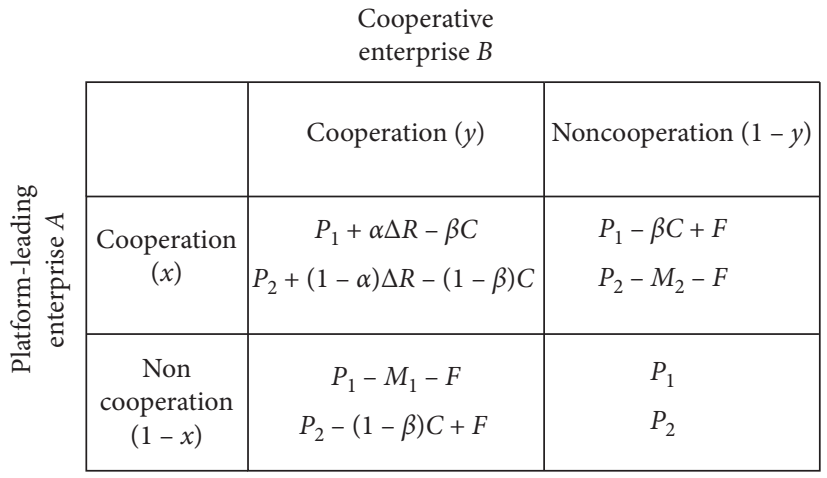

FIgURE 1: The payoff matrix of the two players in the evolutionary game.

TABLE 1: The specifications of variables in the tripartite evolutionary game.

\begin{tabular}{lc}
\hline Variables & Description \\
\hline$E_{a 1}$ & The expected return from the cooperation of enterprise $A$ \\
$E_{a 2}$ & The expected return from the noncooperation of enterprise $A$ \\
$E_{a}$ & The average expected return from the cooperation and noncooperation of enterprise $A$ \\
$E_{b 1}$ & The expected return from the cooperation of enterprise $B$ \\
$E_{b 2}$ & The expected return from the noncooperation of enterprise $B$ \\
$E_{b}$ & The average expected return from the cooperation and noncooperation of enterprise $B$ \\
\hline
\end{tabular}

$$
\begin{aligned}
E_{b 1}= & x\left[P_{2}+(1-\alpha) \Delta R-(1-\beta) C\right] \\
& +(1-x)\left[P_{2}-(1-\beta) C+F\right]=x(1-\alpha) \Delta R+(1-x) F \\
& +P_{2}-(1-\beta) C, \\
E_{b 2}= & x\left(P_{2}-M_{2}-F\right)+(1-x) P_{2}=P_{2}-x M_{2}-x F, \\
E_{b}= & y E_{b 1}+(1-y) E_{b 2}=x y(1-\alpha) \Delta R+P_{2}-x(1-y) M_{2} \\
& -(1-\beta) y C .
\end{aligned}
$$

The replication dynamic equations of both $A$ and $B$ are as

$$
\begin{aligned}
& \frac{\mathrm{d} x}{\mathrm{~d} t}=x\left(E_{a 1}-E_{a}\right)=x(1-x)\left[E_{a 1}-E_{a 2}\right]=x(1-x)\left[y\left(\alpha \Delta R+M_{1}\right)+F-\beta C\right], \\
& \frac{\mathrm{d} y}{\mathrm{~d} t}=y\left(E_{b 1}-E_{b}\right)=y(1-y)\left[E_{b 1}-E_{b 2}\right]=y(1-y)\left\{x\left[(1-\alpha) \Delta R+M_{2}\right]+F-(1-\beta) C\right\} .
\end{aligned}
$$

(2) Evolutionary Stable Strategies for A. If $(\mathrm{d} x / \mathrm{d} t)=0$, there are three stable solutions to equation (3), $x_{1}^{*}=1, x_{2}^{*}=0$, and $x_{3}^{*}=\left((\beta C-F) /\left(\alpha \Delta R+M_{1}\right)\right)$.

If formula (3) is a derivative and $\left(\mathrm{d}^{2} x / \mathrm{d}^{2} t\right)=0$, then the formula can be obtained as follows:

$$
\frac{\mathrm{d}^{2} x}{\mathrm{~d}^{2} t}=(1-2 x)\left[y\left(\alpha \Delta R+M_{1}\right)+F-\beta C\right] .
$$

Next, we discuss the stable solutions of $A$ under different probabilities in three cases.

(a) When $y>\left((\beta C-F) /\left(\alpha \Delta R+M_{1}\right)\right), x_{1}^{*}=1$ is a stable solution, which means that platform-leading enterprise $A$ will choose to cooperate with relevant enterprise $B$. The evolution results are shown in Figure 2.

(b) When $y<\left((\beta C-F) /\left(\alpha \Delta R+M_{1}\right)\right), x_{2}^{*}=0$ is a stable solution, which means that platform-leading enterprise $A$ will choose noncooperation. The evolution results are shown in Figure 3.

(c) When $y=\left((\beta C-F) /\left(\alpha \Delta R+M_{1}\right)\right), \quad(\mathrm{d} x / \mathrm{d} t)=0$, which means that any point is stable. The evolution results are shown in Figure 4.

(3) Evolutionary Stable Strategies for B. If $(\mathrm{d} y / \mathrm{d} t)=0$, there are three stable solutions to equation (4), $y_{1}^{*}=1, y_{2}^{*}=0$, and $y_{3}^{*}=\left(((1-\beta) C-F) /\left((1-\alpha) \Delta R+M_{2}\right)\right)$. There are still 


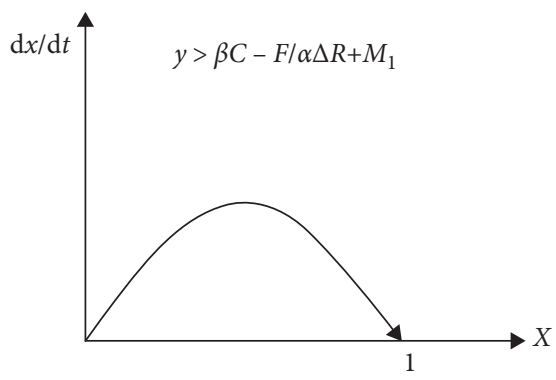

FIGURE 2: Stable solution for $y>\left((\beta C-F) /\left(\alpha \Delta R+M_{1}\right)\right)$.

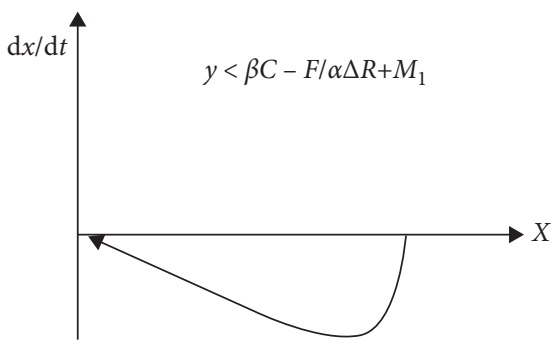

Figure 3: Stable solution for $y<\left((\beta C-F) /\left(\alpha \Delta R+M_{1}\right)\right)$.

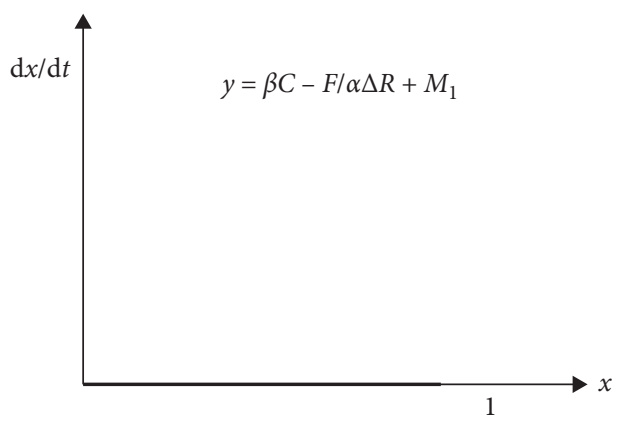

Figure 4: Stable solution for $y=\left((\beta C-F) /\left(\alpha \Delta R+M_{1}\right)\right)$.

three cases for discussing the stable solution of B under different probabilities.

(a) When $x>\left(((1-\beta) C-F) /\left((1-\alpha) \Delta R+M_{2}\right)\right), y_{1}^{*}=$ 1 is a stable solution, which means that enterprise $B$ will choose a strategy of cooperation. The evolution results are shown in Figure 5.

(b) When $\quad x<\left(((1-\beta) C-F) /\left((1-\alpha) \Delta R+M_{2}\right)\right)$, $(\mathrm{d} y / \mathrm{d} t)>1 . y_{2}^{*}=0$ is a stable solution, which means that enterprise $B$ will choose noncooperation. The evolution results are shown in Figure 6.

(c) When $\quad x=\left(((1-\beta) C-F) /\left((1-\alpha) \Delta R+M_{2}\right)\right)$, $(\mathrm{d} y / \mathrm{d} t)=0$, which means that any point is stable. The evolution results are shown in Figure 7.

(4) Combination Evolutionary Stable Strategies for Both A and $B$. We analyze the combination evolution strategies of $A$ and $B$. First, we analyze the responses of $B$ when $A$ adopts different strategies.

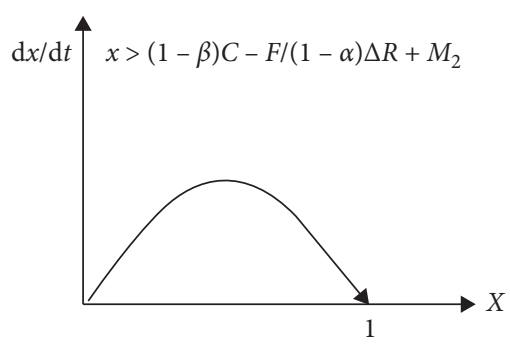

Figure 5: Stable solution for $x>(((1-\beta) C-F) /((1-\alpha) \Delta R+$ $\left.M_{2}\right)$ ).

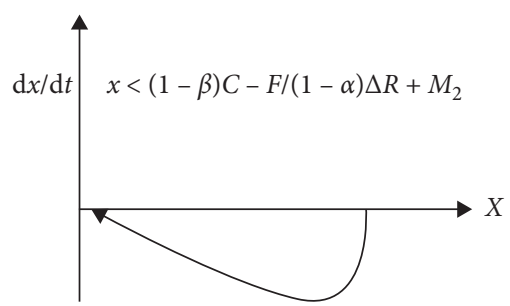

FIgURE 6: Stable solution for $x<(((1-\beta) C-F) /((1-\alpha) \Delta R+$ $\left.M_{2}\right)$ ).

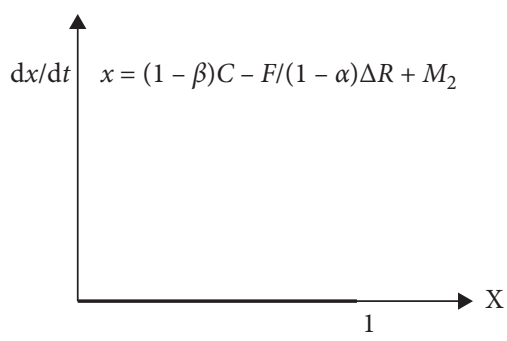

Figure 7: Stable solution for $x=\left((1-\beta) C-F /(1-\alpha) \Delta R+M_{2}\right)$.

(1) When $\left((\beta C-F) /\left(\alpha \Delta R+M_{1}\right)\right)<0, \quad y>((\beta C-F) /$ $\left.\left(\alpha \Delta R+M_{1}\right)\right)$, which means that no matter what $B$ 's reaction is, $A$ will choose a cooperative strategy without hesitation.

(2) When $\left((\beta C-F) /\left(\alpha \Delta R+M_{1}\right)\right)=0, y=0$. Any value of $X$ is a stable solution, which means there is no special motivation for $A$ to choose cooperation or noncooperation.

(3) When $\left((\beta C-F) /\left(\alpha \Delta R+M_{1}\right)\right)>1, \quad y<((\beta C-F) /$ $\left.\left(\alpha \Delta R+M_{1}\right)\right)$. No matter how $B$ reacts, $A$ will adopt a strategy of noncooperation.

Similarly, let us analyze the responses of $A$ when $B$ adopts different strategies.

(4) When $\left.\left(((1-\beta) C-F) /\left((1-\alpha) \Delta R+M_{2}\right)\right)<0, \quad x\right\rangle$ $\left(((1-\beta) C-F) /\left((1-\alpha) \Delta R+M_{2}\right)\right)$. This means that no matter what $A$ 's response is, $B$ will only adopt a cooperative strategy.

(5) When $\left(((1-\beta) C-F) /\left((1-\alpha) \Delta R+M_{2}\right)\right)=0, \quad x=$ 0 . Any value of $y$ is a stable solution, which means that $B$ has no particular incentive to cooperate or not to cooperate because both results are similar for $B$. 
(6) When $\quad\left(((1-\beta) C-F) /\left((1-\alpha) \Delta R+M_{2}\right)\right) \geq 1$, $x<\left(((1-\beta) C-F) /\left((1-\alpha) \Delta R+M_{2}\right)\right)$. This means that no matter how $A$ reacts, $B$ only adopts a strategy of noncooperation.

The results of these comprehensive games are shown in Figure 8.

In Figure 8, there are five equilibrium points, which are $O(0,0), B(1,1), A(1,0), C(0,1)$, and $Q(((\beta C-F) /(\alpha \Delta R+$ $\left.\left.\left.M_{1}\right)\right),\left(((1-\beta) C-F) /\left((1-\alpha) \Delta R+M_{2}\right)\right)\right)$.

In the region $O A Q C$, the result of the game evolution tends to be $O(0,0)$. This means that both sides choose a noncooperative strategy. In the region $A B C Q$, both sides tend to choose cooperative strategies; then, $B(1,1), A(1,0)$, and $C(0,1)$ are unstable because if one party does not cooperate, there will be no stable equilibrium point. $Q(((\beta C-$ $\left.\left.F) /\left(\alpha \Delta R+M_{1}\right)\right),\left(((1-\beta) C-F) /\left((1-\alpha) \Delta R+M_{2}\right)\right)\right)$ is the saddle point.

Therefore, the evolutionary game strategy is that both sides choose to cooperate or both sides choose not to cooperate. The combination of one party cooperation and the other party noncooperation is unstable. Whether the two parties choose to cooperate at the same time or not depends on the location of the saddle point $Q$. The closer the $Q$ point is to the $B$ point, the higher the probability of both parties choosing cooperation. The closer the $Q$ point is to the $O$ point, the higher the probability of noncooperation.

\section{Factors Influencing the Result of the Game}

The position of point $Q$ is observed, which is composed of two triangles, $\triangle O Q C$ and $\triangle O Q A$. Therefore, the discussion of the position of point $Q$ can be transformed into the discussion of quadrilateral $\square O A Q C$ :

$$
S_{\square \mathrm{OAQC}}=S_{\triangle \mathrm{OQC}}+S_{\triangle \mathrm{OQA}}=\frac{1}{2}\left[\frac{\beta C-F}{\alpha \Delta R+M_{1}}+\frac{(1-\beta) C-F}{(1-\alpha) \Delta R+M_{2}}\right] \text {. }
$$

The evolution results of the three situations are discussed as follows:

(A) When $S_{\square \mathrm{OAQC}}>S_{\square \mathrm{QABC}}$, it evolves to $O$, and neither side cooperates

(B) When $S_{\square \mathrm{OAQC}}<S_{\square \mathrm{QABC}}$, it evolves to $B$, and both sides cooperate

(C) When $S_{\square \mathrm{OAQC}}=S_{\square \mathrm{QABC}}$, there is an equal probability of it evolving towards $O$ or $B$

We can understand the influence of each factor on evolution in more detail through calculus derivation.

(1) Excess distribution coefficient $\alpha$

$$
\frac{\mathrm{d} s}{\mathrm{~d} \alpha}=\frac{1}{2}\left\{\frac{(1-\beta) C-F}{\left[(1-\alpha) \Delta R+M_{2}\right]^{2}}-\frac{\beta C-F}{\left(\alpha \Delta R+M_{1}\right)^{2}}\right\},
$$

where $\left(\mathrm{d}^{2} s / \mathrm{d}^{2} \alpha\right)>0$ when $\alpha$ solves the second derivative, and $S_{\square \mathrm{OAQC}}$ has a minimum value.

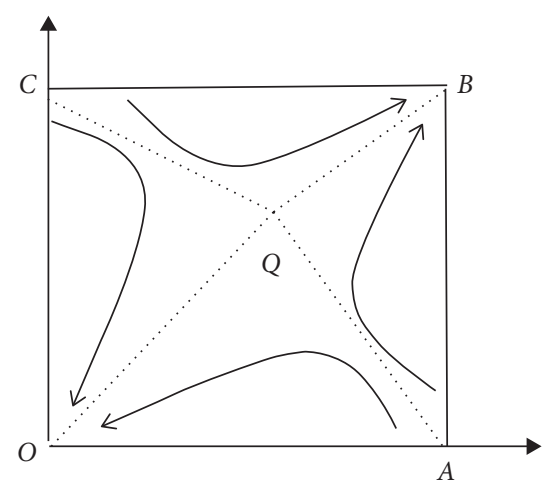

FIgURE 8: Comprehensive game process of $A$ and $B$.

Therefore, when $\alpha$ is large, the evolutionary direction is that both sides adopt cooperative strategies.

(2) Excess return $\Delta R$

$$
\frac{\mathrm{d} s}{\mathrm{~d} \Delta R}=-\frac{1}{2}\left\{\frac{\alpha(\beta C-F)}{\left(\alpha \Delta R+M_{1}\right)^{2}}+\frac{(1-\alpha)[(1-\beta) C-F]}{\left[(1-\alpha) \Delta R+M_{2}\right]^{2}}\right\}<0,
$$

where $(\mathrm{d} s / \mathrm{d} \Delta R)$ is a minus function and $\Delta R$ and $S_{\square \mathrm{OAQC}}$ change in reverse. That is to say, the larger the value of $\Delta R$ is, the stronger the willingness of both sides to cooperate and create value together is.

(3) Imitation costs $M_{1}$ and $M_{2}$

$$
\begin{aligned}
& \frac{\mathrm{d} s}{\mathrm{~d} M_{1}}=-\frac{1}{2}\left[\frac{(\beta C-F)}{\left(\alpha \Delta R+M_{1}\right)^{2}}\right], \\
& \frac{\mathrm{d} s}{\mathrm{~d} M_{2}}=-\frac{1}{2}\left\{\frac{(1-\beta) C-F}{\left[(1-\alpha) \Delta R+M_{2}\right]^{2}}\right\} .
\end{aligned}
$$

This shows that if the cost of "piracy" technology is very low for technology imitators, they will adopt a noncooperative strategy, which is not conducive to the evolution of an innovation ecosystem. If the cost of "piracy" technology is high, they will cooperate with platform-leading enterprises.

(4) Cost-sharing coefficient $\beta$

$$
\frac{\mathrm{d} s}{\mathrm{~d} \beta}=\frac{1}{2}\left[\frac{C}{\alpha \Delta R+M_{1}}-\frac{C}{(1-\alpha) \Delta R+M_{2}}\right] .
$$

When $\quad\left(C /\left(\alpha \Delta R+M_{1}\right)\right)<\left(C /\left((1-\alpha) \Delta R+M_{2}\right)\right)$, $(\mathrm{d} s / \mathrm{d} \beta)$ is a minus function. In this case, the greater the value of $\beta$ is, the less likely the evolution of mutual cooperation will be.

When $\quad\left(C /\left(\alpha \Delta R+M_{1}\right)\right)>\left(C /\left((1-\alpha) \Delta R+M_{2}\right)\right)$, $(\mathrm{d} s / \mathrm{d} \beta)$ is a monotone-increasing function. In this case, the larger the value of $\beta$ is, the more noncooperative the two sides will evolve to be. 
(5) Coordination costs $C$

$$
\frac{\mathrm{d} s}{\mathrm{~d} C}=\frac{1}{2}\left[\frac{\beta}{\alpha \Delta R+M_{1}}+\frac{1-\beta}{(1-\alpha) \Delta R+M_{2}}\right]>0,
$$

which indicates that $(\mathrm{d} s / \mathrm{d} C)$ is a monotone-increasing function. This means that, with increasing cooperation costs, both parties will be more willing to adopt the strategy of noncooperation. If the costs of cooperation are reduced, both sides will be more willing to cooperate.

(6) Penalties $F$

$$
\frac{\mathrm{d} s}{\mathrm{~d} F}=-\frac{1}{2}\left[\frac{1}{\alpha \Delta R+M_{1}}+\frac{1}{(1-\alpha) \Delta R+M_{2}}\right]<0,
$$

which indicates that $(\mathrm{d} s / \mathrm{d} F)$ is a monotone-decreasing function. The smaller $F$ is, the larger $S_{\square O A Q C}$ is and the more noncooperative the two sides are, so the degree of the penalty also affects cocreation in the ecosystem. Fewer penalties will increase the free riding of technology and the "piracy" of technology, which will affect the cooperation mechanism. High penalties and the effective protection of technology can promote cooperation and cocreation in the ecosystem.

\section{Case Analysis of an AI Enterprise: Taking iFLYTEK as an Example}

According to the model of game theory, we took a leading Chinese AI enterprise, iFLYTEK, as an example to verify the hypothesis. iFLYTEK was founded in 1999. Following years of development, iFLYTEK has many world-class patents in speech recognition, speech synthesis, and other fields. In 2008, iFLYTEK was listed in the $A$-share market. iFLYTEK released the "iFLYTEK Voice Cloud Platform" in 2010 and launched the "iFLYTEK Super Brain Plan ("Super Brain Plan" is a major forward-looking project for advanced artificial intelligence (i.e., cognitive intelligence) initiated by iFLYTEK. It is also a key project supporting iFLYTEK's goal of "making computers understand and think" on the basis of "making computers listen and speak")" in 2014 [44]. The human-computer interface AIUI (AIUI is a new generation of human-computer intelligent interactive open platform of iFLYTEK, which aims to realize the barrier-free interaction between human and machine and make the communication between human and machine natural through voice, image, gesture, and other ways) was released in 2015. After many years, iFLYTEK has become the absolute leader in the field of intelligent voice. iFLYTEK is now a firm leader in China, accounting for more than $60 \%$ of the market share in the intelligent speech industry and more than $70 \%$ in the speech synthesis market (source: China securities net. http:// stockdata.cnstock.com/stock/sz002230.html). Table 2 shows the development of iFLYTEK.
The establishment of the iFLYTEK voice ecosystem has gone through three stages. iFLYTEK's initial stage was from 1999 to 2003. In 1999, during the wave of entrepreneurship among Chinese college students, Qingfeng Liu, the founder of iFLYTEK, started to implement the idea of commercializing the intelligent voice products. However, in the early days of entrepreneurship, iFLYTEK experienced the dilemmas of poor product sales and difficult operations. Under great pressure to survive, the founding team held a meeting in Chaohu, Anhui Province to discuss the strategic development of the company in the future, which was also an opportunity to change the fate of iFLYTEK. After the conference, iFLYTEK seized the huge business opportunity offered by China's call-center market and created value together with telecom operators and external partners (including key customers and international leading intelligent voice companies). iFLYTEK first chose to occupy the market through external cooperation (with Nuance, an international voice provider specializing in speech recognition) and began to provide services for Huawei and other enterprises. In the second stage of growth, iFLYTEK seized the new business opportunity offered by $3 \mathrm{G}$, developed new products, cooperated deeply with the education industry, and actively promoted the business of voice technology in oral examinations. In 2008, iFLYTEK entered the capital market and was listed on Shenzhen Stock Exchange, with a revenue of RMB 257 million yuan in the same year (http://stock. hexun.com/2009-01-15/113455316.html). In its maturity stage, iFLYTEK realized its transformation from a single voice provider to an ecological voice platform. During the wave of AI, iFLYTEK adjusted its strategy to become the pioneer of the AI industry, and its "Super Brain Plan" came into being against this backdrop. iFLYTEK focuses on practical product functions, such as calling, texting, and listening to music. At the same time, with the development of AI technology, the "iFLYTEK Voice Cloud" has evolved into the "iFLYTEK Open Platform" and has formed an integrated voice ecosystem. iFLYTEK encourages employees to incubate new businesses within the company, cooperates with big enterprises, and has penetrated the fields of mobile phones, education, home furnishings, and cars, forming a voice ecosystem. Ranked in the first place in China on the 50 Smartest Companies 2017 list, which was announced by MIT Technology Review (“50 Smartest Companies 2017”, MIT Technology Review, June 27, 2017, https://www. technologyreview.com/lists-tr50/what-are-the-50-smartestcompanies/), iFLYTEK is increasingly influential with its superior voice technology in the AI industry.

iFLYTEK has gradually established a voice innovation ecosystem and cooperated with many enterprises in the system to create value together so as to realize its transformation from a small voice technology provider to a leading voice ecosystem enterprise. iFLYTEK's coordination mechanism of value cocreation and its experience of establishing a voice ecological innovation system are very representative. Its growth path and the puzzles encountered 
TABLE 2: A chronicle of iFLYTEK over the years.

\begin{tabular}{c}
\hline Year \\
\hline $1999 \begin{array}{c}\text { iFLYTEK innovation event } \\
\text { Speech-synthesis evaluation scored more than } 3.0 \\
\text { iFLYTEK undertook the national voice high-technology } \\
\text { industrialization demonstration project and set up a } \\
\text { postdoctoral research workstation }\end{array}$ \\
2004 In the international evaluation of Chinese speech synthesis, \\
iFLYTEK ranked first for all indicators \\
The iFLYTEK Research Institute of Science and Technology was \\
officially established and won the "major technological \\
invention award of information industry"
\end{tabular}

2014 iFLYTEK launched the "iFLYTEK Super Brain Program"

iFLYTEK released the human-computer voice interface,

2015 defining the new standard of human and voice interaction technology in the era of the Internet of Things

iFLYTEK launched "AI education," entered the medical field, and released its "intelligent medical assistant"

iFLYTEK deeply explored the new combinations of voice AI in iFLYTEK business event IFLYTEK was founded

iFLYTEK completed its second round of financing and cooperated with Nuance, which is the world's leading voice company, to provide an automatic response scheme

iFLYTEK achieved profit and loss balance for the first time, and its sales exceeded 100 million yuan for the first time

iFLYTEK's voice product revenue reached 150 million yuan, driving the industry by about 1 billion yuan

iFLYTEK was listed on the Shenzhen Stock Exchange, and the construction of the voice industry base was started, with a revenue of 257 million in 2008

iFLYTEK released "iFLYTEK Voice Cloud" and "Voice Input Method" and established a national intelligent voice high-tech industrial base

iFLYTEK's Voice Cloud end users exceeded 150 million, with it becoming the largest voice-listed company in the Asia Pacific region and the software company with the highest market value in

the Shanghai and Shenzhen stock markets of China ("New generation of 'voice cloud' released by iFLYTEK," NetEase News, March 23, 2012, http://news.163.com/12/0323/12/ 7T9G6BI700014AEE.html)

iFLYTEK established a comprehensive strategic cooperative relationship with the three major telecom operators. iFLYTEK had over 350 million voice cloud downloads and activations and over 100 million voice input users

iFLYTEK released 3.0 smart voice products and the iFLYTEK Voice Cloud, with an annual revenue of 1.77 billion, more than 600 million end users of the iFLYTEK Voice Cloud, and more than 55,000 development cooperation projects

iFLYTEK and JingDong performed strategic cooperation and released the DingDong smart speaker. The total number of users of iFLYTEK's open platform was 700 million, with 300 million users of iFLYTEK's input method and 18 dialects supported (https:// xueqiu.com/2143043140/135269696)

iFLYTEK's open platform had more than 3 billion online daily services, 250,000 partners, and 910 million users (https://www. yicai.com/news/5424180.html). iFLYTEK accounted for more than $60 \%$ of the market share in the field of Chinese speech technology and more than $70 \%$ of the market share in the field of speech synthesis products

2019 the fields of medical treatment, media, education, politics, and law

(Data sources: shown as table $\mathrm{S} 1$ in appendix and sorted by authors)

are the epitome of China's AI enterprises. There are six factors that affect the coordination mechanism of value cocreation of the iFLYTEK voice ecosystem, which will be discussed in following.

5.1. Distribution Coefficient $\alpha$. With the development of Internet technology, iFLYTEK has invested a lot of money to build a developer platform, whatever the cost, sharing more benefits with its partners and sharing relevant technologies with the open platform. iFLYTEK's business philosophy in its innovation ecosystem has been "working together to share industrial achievements," always adhering to the principle of not directly competing with partners and sincerely cooperating with many partners in the ecosystem to jointly promote the rapid growth of the voice industry. For example, iFLYTEK continues to reduce the threshold of innovation and promote applications in the field of voice interaction technology. In 2010, before Echo and Alexa were launched, iFLYTEK launched its first Chinese voice developer platform, the "iFLYTEK Voice Cloud," which provides partners with free speech recognition technology. The construction of this intelligent voice platform had a win-win effect: for entrepreneurs, huge investments in servers were 
avoided, and for iFLYTEK, a broad market for small- and medium-sized enterprises with strong growth potential was obtained. During this period, Tencent QQ, Gaode, Ctrip, and other Internet companies were iFLYTEK's customers. Just five years later, iFLYTEK AIUI successfully went online, sharing rich open resources, strong customization ability, and complete personalized functions for partners, putting forward new scenarios and concepts of human-computer interaction and using its own source innovation technology to achieve the dreams of more innovative entrepreneurs (Yicai, "iFLYTEK has released a new version of its $\$ 2.1$ billion open-platform AIUI," Baidu, May 18, 2018, http://baijiahao.baidu.com/ $s ? \mathrm{id}=1600781656523033242 \& w f r=$ spider $\&$ for $=p c)$. iFLY TEK has also cultivated a number of enterprises in the voice ecosystem, such as Taoyun Technology Co. Ltd., Yunji Technology Co. Ltd., Lieju Technology Co. Ltd., and Yundong Technology Co. Ltd., through internal entrepreneurship and strategic investment mechanisms.

By distributing more benefits to the partners, iFLYTEK innovation ecosystem has gathered more than 1.12 million AI developers and developed more than 750,000 software applications. In 2019, iFLYTEK's consumer business achieved an operating revenue of 3.625 billion, an increase of $43.99 \%$, and its gross profit was 1.708 billion, an increase of $31.81 \%$ (data is from the iFLYTEK annual report in 2019). Through technology empowerment, market empowerment, and investment empowerment, the iFLYTEK innovation ecosystem provides all-round technical cooperation and operation services for the partners from budding to growing and continues to create a win-win ecosystem.

5.2. Excess Return $\Delta R$. Adhering to the concept of "working together to share industrial achievements," iFLYTEK actively cooperates with universities and scientific research institutions to establish a joint laboratory for cooperative research and development and insists on not competing directly with development partners in terms of cooperation. iFLYTEK and relevant enterprises form strategic alliances to carry out innovation activities together to promote the progress of voice technology in China and realize its industrial development. Table 3 shows the details. In the era of knowledge sharing, based on iFLYTEK's technology innovation platform, each cooperative enterprise has promoted the formation and evolution of the intelligent voice industry innovation ecosystem, which is "market-oriented, enterprise-oriented, and industry university research-combined." In this process, iFLYTEK always plays the role of the core enterprise and has a key role in the development of the innovation ecosystem of the intelligent voice industry in China.

In the cooperation with partners, iFLYTEK has always maintained the dominant position in the ecosystem and designed a mutually beneficial mechanism. By 2019, the iFLYTEK ecosystem has 1.6 million ecological partners. Due to the numerous partners in the ecosystem, iFLYTEK has made an in-depth layout in education, consumer, medical, urban management, and other industries. iFLYTEK has established a nationwide marketing channel and service network. For example, in the field of education, aiming at the market demand of personalized learning, iFLYTEK has cooperated with partners in the ecosystem to deeply tap the value of data and helped schools improve the effectiveness of students' learning. Its products have covered more than 16,000 schools across the country. In the field of intelligent medicine, iFLYTEK intelligent medical assistant of HKUST has provided 25 million times of auxiliary diagnosis suggestions, with an average of more than 200,000 pieces of data per day, serving more than 30,000 grassroots' doctors and benefiting more than 40 million residents. In terms of smart city governance, iFLYTEK has built a new AI capability platform "City Super Brain" and built urban smart applications around urban governance, so as to realize scientific government decision-making, fine social governance, and efficient public service. The relevant products have been launched in nearly 30 cities in 10 provinces in China (data is from the iFLYTEK annual report in 2019).

5.3. Imitation Costs $M_{1}$ and $M_{2}$. A big risk faced by many technology-based companies in the initial stage is that their core technology is imitated by competitors, and the cost of this imitation is much lower, so they lose competitiveness. For example, iFLYTEK builds the Intelligent Voice Industry Alliance Center to increase the imitation cost of competitors in the early stage. In March 2000, iFLYTEK cooperated with a large number of voice experts and scientific research institutes to launch the project "Voice Entrepreneurship Alliance in China." iFLYTEK has established the knowledge cooperation network with nearly 100 units including China University of Science and Technology, Institute of Acoustics of Chinese Academy of Sciences, Institute of Automation of Chinese Academy of Sciences, Institute of Language of Chinese Academy of Social Sciences, Huawei, IBM, and Intel and jointly established the joint laboratory, namely, "iFLYTEK Alliance Center" [45]. In 2014, the "Super Brain Program" was launched to attract the world's top voice experts to join in (annual speech of the head of the internal team of iFLYTEK "Super Brain Project," [2016-02-01], https://www.sohu.com/a/57533728_336009). In 2016, the core research platform was established, and the humancomputer interaction AIUI system was developed, which expanded AI technology to the fields of smart cities, education, and finance. Secondly, iFLYTEK increases the imitation cost of competitors, which is to use the patent map to monitor the technology trends of competitors. The patent map is a kind of patent analysis and research method. It processes and analyzes patent information, reflects the information hidden in patent data through various interpretable charts, number of patents, annual growth chart, etc., so as to analyze the technology distribution situation and provide more intuitive information for decision makers. iFLYTEK uses the patent map to monitor the technology dynamics of competitors, so as to guide iFLYTEK's technology development and strategic layout and effectively prevent competitors from imitation. iFLYTEK sets a high technical threshold, which was difficult for rivals to imitate. 
TABLE 3: iFLYTEK's cooperation events.

\begin{tabular}{|c|c|c|c|}
\hline Year & Partners & Cooperation project & Excess returns from cooperation \\
\hline 2000 & Huawei, IBM, Intel, Analog, etc. & $\begin{array}{c}\text { Establishment of the iFLYTEK Alliance } \\
\text { Center }\end{array}$ & $\begin{array}{l}\text { Partners obtained voice service products. } \\
\text { iFLYTEK held the main voice market share }\end{array}$ \\
\hline 2006 & $\begin{array}{c}\text { Multimedia Signal and Intelligent } \\
\text { Information Processing Laboratory, } \\
\text { Department of Electronic Engineering, } \\
\text { Tsinghua University }\end{array}$ & $\begin{array}{l}\text { Joint establishment of Tsinghua } \\
\text { iFLYTEK Speech Technology Joint } \\
\text { Laboratory }\end{array}$ & $\begin{array}{l}\text { Tsinghua-iFLYTEK cooperation achieved } \\
\text { major technological breakthroughs in speech } \\
\text { recognition, audio content analysis, speech } \\
\text { retrieval, language understanding, data } \\
\text { mining, and other fields }\end{array}$ \\
\hline 2011 & $\begin{array}{c}\text { University of Science and Technology of } \\
\text { China }\end{array}$ & $\begin{array}{l}\text { Establishment of National Engineering } \\
\text { Laboratory for Intelligent Speech and } \\
\text { Language Information Processing }\end{array}$ & $\begin{array}{c}\text { Cooperation accelerated technological } \\
\text { breakthroughs in human-computer } \\
\text { interaction, AI, and massive information } \\
\text { processing }\end{array}$ \\
\hline 2012 & $\begin{array}{c}\text { Cooperation with } 19 \text { companies, including } \\
\text { Huawei, Lenovo, China Mobile, China } \\
\text { Telecom, and China Unicom }\end{array}$ & $\begin{array}{l}\text { Joint establishment of the China Speech } \\
\text { Industry Alliance }\end{array}$ & $\begin{array}{c}\text { Cooperation integrated industrial resources, } \\
\text { building a healthy industrial ecosystem, and } \\
\text { promoting the development of the Chinese } \\
\text { speech industry }\end{array}$ \\
\hline 2014 & Harbin Institute of Technology & $\begin{array}{l}\text { Joint establishment of "Harbin Institute } \\
\text { of Technology-iFLYTEK Cognitive } \\
\text { Language Computing Joint Laboratory" }\end{array}$ & $\begin{array}{c}\text { The cooperation strengthened long-term and } \\
\text { in-depth research in the field of cognitive } \\
\text { language computing }\end{array}$ \\
\hline 2015 & York University & $\begin{array}{c}\text { Establishment of the iFLYTEK Neural } \\
\text { Computing and Deep Learning } \\
\text { Laboratory }\end{array}$ & $\begin{array}{l}\text { Cooperation promoted breakthroughs in } \\
\text { neural computing, deep learning, and AI } \\
\text { technology }\end{array}$ \\
\hline 2018 & MIT Computer Science and AI Lab & AI Alliance & $\begin{array}{c}\text { Cooperation in speech processing, reasoning, } \\
\text { cognition, and AI }\end{array}$ \\
\hline
\end{tabular}

It is also an important factor for iFLYTEK becoming a leader in the voice ecosystem.

5.4. Cost-Sharing Coefficient $\beta$. With the arriving of mobile Internet, iFLYTEK realized that this would be a huge business opportunity. At the same time, iFLYTEK received application demands from small- and medium-sized enterprises and developers. Before that, iFLYTEK's customers were mainly large- and medium-sized enterprises, and the development costs were too high. After turning to the market of small- and medium-sized enterprises, the coordination costs were able to be greatly reduced. In 2010, iFLYTEK released the world's first "iFLYTEK Voice Cloud Platform" for mobile Internet intelligent voice interaction and, simultaneously, released the "Voice Input Method" experience version to re-enter the consumer market. The launch of this platform greatly reduced the coordination cost between the partners in the voice ecosystem. For entrepreneurs, the products can be developed directly based on iFLYTEK's Voice Cloud Platform and can provide services to the outside world, reducing huge investment on the computer server. For iFLYTEK, it not only provides a "Voice Cloud Platform" for small- and mediumsized enterprises but also explores a new market for itself. Through the voice ecosystem, iFLYTEK has developed more than 50,000 partners in recent years, and iFLYTEK is also transforming from a single core technology provider to an open-platform enterprise. IFLYTEK's voice innovation ecosystem not only supports the entrepreneurial team but also cooperates with Ctrip, Sina Weibo, and other large companies to develop APPs. While spreading voice interaction functions, iFLYTEK has also won a wide range of users. iFLYTEK directly obtains income through cooperation with large companies, and after supporting small- and medium-sized companies to a certain scale, it will also share the income with them, thus generating economic benefits for iFLYTEK. iFLYTEK plays an important role in the voice ecosystem, leads the key process of the value chain, and constantly creates more new technologies. The reduction of the cooperation cost has also improved iFLYTEK's operating income and earnings' per share. Figure 9 shows iFLYTEK's net profit from 2008 to 2019.

5.5. Cooperation Costs $C$. When the intelligent voice market was not mature, iFLYTEK resolutely focused on business-tobusiness (B2B) application scenarios, grasping the needs of $B$-end users with an open mind and accumulating technical resources. For example, Huawei, ZTE, Digital China, and other domestic smart networks, call centers, and business systems needed to use voice engines to buy iFLYTEK. iFLYTEK provided the core competence of voice to partners at a lower cost, and the partners performed specific applications, which not only helped the partners reduce their R\&D costs but also gave iFLYTEK more business opportunities. This was the establishment of the "iFLYTEK Inside" model. When the intelligent voice market was experiencing explosive growth, the industry competition was gradually increasing. iFLYTEK was determined to develop the platform and use cloud technology. Based on the needs of smalland medium-sized enterprises, it continued to iterate the voice recognition algorithm. For small- and medium-sized enterprises, the use of the "iFLYTEK Voice Platform" can significantly reduce huge investments in the server. For iFLYTEK, it has gained a broader voice cloud market for 


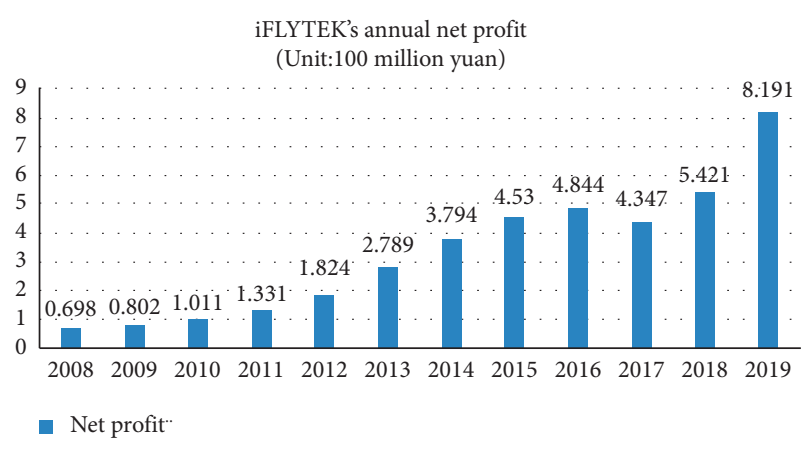

FIGURE 9: iFLYTEK's annual net profit (the data in Figure 9 are compiled by the author according to iFLYTEK's annual reports over the years).

small- and medium-sized enterprises. By reducing the cost of cooperation, leading enterprises and cooperative enterprises can closely strengthen cooperation.

In order to reduce the cooperation cost in the ecosystem, iFLYTEK has established a layout in three aspects. Firstly, iFLYTEK has cooperated with universities and research institutes extensively. The research institute is responsible for the applied technology research of products, while iFLYTEK is responsible for turning laboratory achievements into products. Secondly, iFLYTEK is also actively building an innovative industrial platform. On the one hand, it took the opportunity of the "National 863 Plan Achievements' industrialization base" in May 2000. Through the organization of capital, market, talent, and other resources, the industrial operation support platform is constructed, and the technical advantages are rapidly transformed into industrial advantages. On the other hand, iFLYTEK enables domestic and foreign enterprises to create new products on the basis of the iFLYTEK voice ecosystem and applies voice technology to various industries, so as to realize all-round penetration of the voice market. Thirdly, iFLYTEK has built the voice ecosystem, which has attracted leading enterprises in various industries to join in. iFLYTEK has also penetrated into the application fields of various industries, providing a variety of voice products. There are more than 1500 cooperative development partners in the ecosystem, including not only leading domestic enterprises such as Lenovo, Huawei, and Haier but also international IT giants such as Intel, Epson, and Siemens VDO.

5.6. Penalties $F$. The protection of intellectual property rights is very important for AI enterprises. Patent protection exists to protect the core competitiveness of product research. iFLYTEK conducts wide property rights' exploration and the monitoring of important businesses, adds intellectual property protection to the assessment system, and formulates incentive measures for protection. iFLYTEK has established a patent monitoring and early warning system for competitors. According to the analysis results, the enterprise is divided into three levels of early warning: red, orange, and blue. If the patent layout and technology direction are influenced, the red patent warning is issued urgently, and relevant technical managers are called together to discuss the response plan, so as to flexibly adjust the patent strategy. If there is an obvious but not serious impact on the enterprise, the orange patent warning can be issued, and the infringement situation can be reported to the intellectual property department. If the patent status is normal, the system will detect and display a blue warning. The patent report is published quarterly or biannually. iFLYTEK has established a set of patent monitoring and early warning system to punish imitators and ensure that its dominant market position can be maintained. Using the exclusive advantage of patent to punish imitators, IFLYTEK can use patent litigation to prohibit the sale of products of defaulting enterprises, so as to protect the sound ecosystem.

\section{Discussion}

6.1. Conclusions. Based on EGT, this paper constructs the value cocreation mechanism of the innovation ecosystem of an $\mathrm{AI}$ enterprise and discusses the factors that affect such value cocreation. Taking iFLYTEK as an example, China's leading AI enterprise, six factors are verified that affect the value cocreation in the ecosystem. The results show that obtaining an excess return rate, the distribution coefficient of the excess return rate, coordination costs, the cost-sharing coefficient, imitation costs, and penalties will affect value cocreation in an innovation ecosystem. The main conclusions are as follows:

First, the cooperation mechanism of leading enterprises in an $\mathrm{AI}$ ecosystem depends on the position of the saddle point. By establishing the replication dynamic equation, the stable evolutionary strategy is found. The evolution results show that there are only two stable results of the game: both sides choose to cooperate or not to cooperate. The combination of one party cooperating and the other not cooperating is unstable. Whether the two sides choose to cooperate or not at the same time depends on the location of the saddle point. The closer the saddle point is to $(1,1)$, the higher the probability of both sides choosing cooperation. The closer the saddle point is to $(0,0)$, the higher the probability of noncooperation.

Second, the location of the saddle point is determined by six factors, which are the excess distribution coefficient, excess returns, imitation costs, the cost-sharing coefficient, coordination costs, and penalties. The higher the values of the excess distribution coefficient, the excess returns, the imitation costs, the cost-sharing coefficient, and the penalties, the more likely leading enterprises and partners are to cooperate. The lower the value of the coordination costs, the higher the probability of both sides' cooperation.

Third, the paper analyzes the establishment process of the iFLYTEK innovation ecosystem and the value cocreation mechanism at different stages of iFLYTEK's growth, and it then discusses the role of six factors in iFLYTEK's ecosystem. For example, regarding the distribution coefficient, iFLYTEK adheres to the principle of not directly competing with partners and sincerely cooperates with many partners in the ecosystem to jointly promote the rapid growth of the voice industry. Concerning excess returns, iFLYTEK and relevant enterprises form a strategic alliance to carry out innovation activities together to promote the progress of voice technology in China and realize its industrial development. With regard to imitation costs, iFLYTEK set a high technical threshold and made it difficult for rivals to imitate 
its technology. In terms of the cost-sharing coefficient, the launch of the "iFLYTEK Voice Cloud Platform" project has greatly reduced the coordination costs between the partners in the voice ecosystem. Concerning cooperation costs, iFLYTEK provides the core competence of voice to its partners at a lower cost, and the partners perform the specific applications, which not only helps the partners reduce their $R \& D$ costs but also provides iFLYTEK with more business opportunities. With regard to penalties, iFLYTEK conducts wide property rights exploration, adds intellectual property protection to the assessment system, and formulates incentive measures for protection.

6.2. Contributions. The significant theoretical contributions of this work are threefold. First, our findings contribute to AI research by providing new insights through the discussion of dynamic cooperation mechanisms in AI innovation ecosystems. By solving the equilibrium solution of a replication dynamic equation, the paper analyzes the dynamic game process between leading AI enterprises and partners. There are two kinds of equilibrium strategies in the evolutionary game, either both parties cooperate or both parties do not cooperate. The combination of one party cooperating and the other party not cooperating is unstable. Whether the two sides cooperate depends on the location of the saddle point. Our research expands the application of innovation ecosystems in AI enterprises and explains the dynamic equilibrium process of AI enterprise value cocreation through EGT.

Second, the value cocreation mechanism of an AI enterprise is constructed, and the six factors affecting value cocreation are also discussed. Previous studies mainly focused on AI technology, but less on the cooperation mechanism between AI enterprises. The research in this paper is helpful in terms of opening the "black box" of the value cocreation mechanism and discussing the influencing factors in different situations for the growth of AI ecosystems.

Third, combined with the case study, the paper discusses how AI's leading enterprises and partners can create value together in order to obtain maximum benefits. The paper takes iFLYTEK as an example to illustrate the six factors that influence the cooperation of AI enterprises and to explain how to establish a suitable cocreation mechanism between leading enterprises and partners.

6.3. Managerial Implications. This study provides managerial implications for AI enterprises in terms of formulating strategies for participating in AI ecosystems. First, it is very important to find suitable partners and establish long-term cooperation mechanisms in an AI ecosystem. Most of the founding teams of AI enterprises have a certain technical threshold, but it also leads to the misunderstanding of relying too much on technology and ignoring the market. From technology to product commercialization to industrialization, the process has a long way to go. Therefore, we should break down the narrow understanding of "technology-based independence" and establish a broad cooperation mechanism. In the coordination mechanism of value cocreation, the choice of partner selection is very important. The key to long-term cooperation between ecological partners in the ecosystem is to find suitable partners who can complement each other, share risks and benefits, improve the distribution mechanism of benefits, institutionalize the proportion of excess income distribution, and establish reasonable standards. Value cocreation can promote the healthy development of an innovation ecosystem and benefit all the members of such a system. The basis of value cocreation is a reasonable cooperation mechanism and partner selection criteria.

Second, platform-leading enterprises play a key role in the development of innovation ecosystems, especially at the key moment of system development. Partners can participate in the ecosystem by reducing their cooperation costs. The way to reduce the costs of cooperation is based on specialization. In this way, participants only focus on their best business, not only to improve the scale of their core business but also to provide more opportunities for their partners. In this process, platform-leading enterprises need to be cultivated and supported, improving the coordination and service of their partners. Moreover, there is inevitably "hitchhiking" and "technology theft" in cooperation, which affects the sound operation of the system. This requires increasing the punishment and supervision mechanisms for the members involved in the system, strengthening the protection of intellectual property rights, and restraining the opportunistic behavior of the participants. Before cooperation, responsibilities, rights, and interests, restrictions with effective legal contracts and punishments for "technology theft" should be clearly outlined. Only by establishing restraint mechanisms for the sound operation of the system can we form effective protection for all participants and establish a suitable environment for an innovation ecosystem of AI enterprises.

Third, when the development of AI enterprises reaches a certain stage, there are more types of products and a wider range of businesses. It is necessary to optimize the ecosystem effect through value cocreation. For example, iFLYTEK set up an ecological voice platform to realize a huge transformation from a product provider to a platform provider and extensively connected consumers, education, city, finance, and other businesses on this platform. In the further development of innovation ecosystems, leading enterprises' innovation ability and resource allocation ability will help to clarify the synergy among the species involved. Value cocreation can accurately grasp the current and future technological needs, provide products and services with market prospects, improve the stability and innovation efficiency of the ecosystem, and accelerate its maturity.

6.4. Limitations and Future Research. This paper constructs the coordination mechanism of value cocreation in AI enterprise innovation ecosystems based on EGT. However, 
there are still some limitations to be considered in further research.

First, the paper focuses on the ecosystem development of AI enterprises from the perspective of value cocreation. The exit mechanism in innovation ecosystems can be further studied. For example, in the process of ecosystem evolution, the possible reasons for the quits of partners are as follows: inappropriate partners, violation of the original intention of cooperation, and unreasonable distribution of interests. Second, the paper considers value cocreation from the perspective of platform-leading enterprises. Value cocreation can also be considered from the perspective of partners. Third, although iFLYTEK has experienced great success in the field of voice, it has also gone through numerous detours in terms of its growth. The failures of AI enterprises are also worthy of further study. With the emergence of more and more AI enterprises in China, it is of great significance to study how to build an innovation ecosystem to enable the development of AI enterprises.

\section{Data Availability}

The data used to support the findings of this study are included within the article. Also, these data are available from the corresponding author upon request.

\section{Conflicts of Interest}

The authors declare that there are no conflicts of interest regarding the publication of this paper.

\section{Acknowledgments}

This work was supported by Key Project and Common Project of National Natural Science Foundation of China (71772165 and 72032008), Major Research Projects in Philosophy and Social Sciences of the Ministry of Education in China (17JZD018), National Social Sciences Foundation for Young Scholars (15CGL008), National High-Level Talents' Special Support Plan for Youth Talent (W03070173), and Philosophy and Social Science Planning Project of Zhejiang Province, China (21ZJQN03YB).

\section{Supplementary Materials}

The information about iFLYTEK in this paper mainly comes from iFLYTEK's official website, iFLYTEK's annual report, and interviews with iFLYTEK's senior managers by mainstream media. There are also some literature information from China National Knowledge Infrastructure (CNKI) and Web of Science. (Supplementary Materials)

\section{References}

[1] U. Lichtenthaler, "Open innovation: past research, current debates, and future directions," Academy of Management Perspectives, vol. 25, no. 1, pp. 75-93, 2011.

[2] P. Cerka, J. Grigiene, and G. Sirbikyte, "Liability for damages caused by artificial intelligence," Computer Law \& Security Review, vol. 31, no. 3, pp. 376-389, 2015.
[3] A. P. Botha, "A mind model for intelligent machine innovation using future thinking principles," Journal of Manufacturing Technology Management, vol. 30, no. 8, pp. 1250-1264, 2019.

[4] M. Wauters and M. Vanhoucke, "A nearest neighbour extension to project duration forecasting with artificial intelligence," European Journal of Operational Research, vol. 259, no. 3, pp. 1097-1111, 2017.

[5] U. Lichtenthaler, "Substitute or synthesis: the interplay between human and artificial intelligence," Research-Technology Management, vol. 61, no. 5, pp. 12-14, 2018.

[6] H. Faridi, R. Goodwin, R. Lougee, and J. Martin, "Artificial intelligence and the future of flavor innovation," Cereal Foods World, vol. 64, no. 6, 2019.

[7] S. Magistretti, C. Dell'Era, and A. Messeni Petruzzelli, "How intelligent is Watson? Enabling digital transformation through artificial intelligence," Business Horizons, vol. 62, no. 6, pp. 819-829, 2019.

[8] D. Silver, A. Huang, C. J. Maddison et al., "Mastering the game of go with deep neural networks and tree search," Nature, vol. 529, no. 7587, pp. 484-489, 2016.

[9] C. E. Helfat and J. B. Quinn, "Open innovation: the new imperative for creating and profiting from technology," Academy of Management Perspectives, vol. 20, no. 2, pp. 8688, 2006.

[10] S. Schneider and M. Leyer, "Me or information technology? Adoption of artificial intelligence in the delegation of personal strategic decisions," Managerial and Decision Economics, vol. 40, no. 3, pp. 223-231, 2019.

[11] R. Boyd and R. J. Holton, “Technology, innovation, employment and power: does robotics and artificial intelligence really mean social transformation?" Journal of Sociology, vol. 54, no. 3, pp. 331-345, 2018.

[12] S. W. Elliott, "Artificial intelligence, robots, and work: is this time different?" Issues in Science and Technology, vol. 35, no. 1, pp. 40-44, 2018.

[13] H. Fujii and S. Managi, "Trends and priority shifts in artificial intelligence technology invention: a global patent analysis," Economic Analysis and Policy, vol. 58, pp. 60-69, 2018.

[14] M. Hengstler, E. Enkel, and S. Duelli, "Applied artificial intelligence and trust-the case of autonomous vehicles and medical assistance devices," Technological Forecasting and Social Change, vol. 105, pp. 105-120, 2016.

[15] J. F. Moore, "Predators and prey: a new ecology of competition," Harvard Business Review, vol. 71, no. 3, pp. 75-86, 1993.

[16] B. Leten, W. Vanhaverbeke, N. Roijakkers, A. Clerix, and J. Van Helleputte, "IP models to orchestrate innovation ecosystems: IMEC, a public research institute in nano-electronics," California Management Review, vol. 55, no. 4, pp. 51-64, 2013.

[17] J. F. Li and E. Garnsey, "Policy-driven ecosystems for new vaccine development," Technovation, vol. 34, no. 12, pp. 762-772, 2014.

[18] G. Liu and K. Rong, "The nature of the co-evolutionary process," Group \& Organization Management, vol. 40, no. 6, pp. 809-842, 2015.

[19] R. Kapoor and N. R. Furr, "Complementarities and competition: unpacking the drivers of entrants' technology choices in the solar photovoltaic industry," Strategic Management Journal, vol. 36, no. 3, pp. 416-436, 2015.

[20] S. Lee, Y. Nam, S. Lee, and H. Son, "Determinants of ICT innovations: a cross-country empirical study," Technological Forecasting and Social Change, vol. 110, pp. 71-77, 2016. 
[21] B. Spigel, "The relational organization of entrepreneurial ecosystems," Entrepreneurship Theory and Practice, vol. 41, no. 1, pp. 49-72, 2017.

[22] L. Mulazzani, R. Manrique, and G. Malorgio, "The role of strategic behaviour in ecosystem service modelling: integrating Bayesian networks with game theory," Ecological Economics, vol. 141, pp. 234-244, 2017.

[23] H. L. Madsen, "Business model innovation and the global ecosystem for sustainable development," Journal of Cleaner Production, vol. 247, Article ID 119102, 2020.

[24] M. Masucci, S. Brusoni, and C. Cennamo, "Removing bottlenecks in business ecosystems: the strategic role of outbound open innovation," Research Policy, vol. 49, no. 1, pp. 1-17, 2020.

[25] X. Xie and H. Wang, "How can open innovation ecosystem modes push product innovation forward? An fsQCA analysis," Journal of Business Research, vol. 108, pp. 29-41, 2020.

[26] S. L. Vargo and R. F. Lusch, "Institutions and axioms: an extension and update of service-dominant logic," Journal of the Academy of Marketing Science, vol. 44, no. 1, pp. 5-23, 2016.

[27] S. L. Vargo and R. F. Lusch, "Service-dominant logic: continuing the evolution," Journal of the Academy of Marketing Science, vol. 36, no. 1, pp. 1-10, 2008.

[28] O. Alexy, G. George, and A. J. Salter, "Cui bono? The selective revealing of knowledge and its implications for innovative activity," Academy of Management Review, vol. 38, no. 2, pp. 270-291, 2013.

[29] T. Leclercq, I. Poncin, and W. Hammedi, "The engagement process during value co-creation: gamification in new product-development platforms," International Journal of Electronic Commerce, vol. 21, no. 4, pp. 454-488, 2017.

[30] E. Niesten and I. Stefan, "Embracing the paradox of interorganizational value co-creation-value capture: a literature review towards paradox resolution," International Journal of Management Reviews, vol. 21, no. 2, pp. 231-255, 2019.

[31] Y. Su and T. Li, "Simulation analysis of knowledge transfer in a knowledge alliance based on a circular surface radiator model," Complexity, vol. 2020, Article ID 4301489, 27 pages, 2020.

[32] A. D. Wood, C. F. Mason, and D. Finnoff, "OPEC, the seven sisters, and oil market dominance: an evolutionary game theory and agent-based modeling approach," Journal of Economic Behavior \& Organization, vol. 132, pp. 66-78, 2016.

[33] Z. Yang, Y. Shi, and Y. Li, "Analysis of intellectual property cooperation behavior and its simulation under two types of scenarios using evolutionary game theory," Computers \& Industrial Engineering, vol. 125, pp. 739-750, 2018.

[34] A. Hafezalkotob, R. Mahmoudi, E. Hajisami, and H. M. Wee, "Wholesale-retail pricing strategies under market risk and uncertain demand in supply chain using evolutionary game theory," Kybernetes, vol. 47, no. 6, pp. 1178-1201, 2018.

[35] T. Mekki, I. Jabri, A. Rachedi, and M. B. Jemaa, "Vehicular cloud networking: evolutionary game with reinforcement learning-based access approach," International Journal of BioInspired Computation, vol. 13, no. 1, pp. 45-58, 2019.

[36] X. H. Ma and R. Ji, "Research on evolution of regional synergetic cross-border E-commerce ecosystem," Journal of International Trade and Insurance, vol. 20, no. 2, pp. 87-121, 2019.

[37] E. Abiodun and S. Ivan, "Absorptive capacity and innovation: when is it better to cooperate?" Journal of Evolutionary Economics, vol. 24, no. 2, pp. 399-420, 2014.
[38] D. Brice, A. Oliver, and A. Erkko, "Maneuvering in poor visibility: how firms play the ecosystem game when uncertainty is high," Academy of Management Journal, vol. 61, no. 2, pp. 466-498, 2018.

[39] K. R. Ron, "Value creation in innovation ecosystem: how the structure of technological interdependence affects firm performance in new technology generations," Strategic Management Journal, vol. 31, no. 3, pp. 306-333, 2010.

[40] J. C. Sheng, W. H. Zhou, and B. Z. Zhu, "The coordination of stakeholder interests in environmental regulation: lessons from China's environmental regulation policies from the perspective of the evolutionary game theory," Journal of Cleaner Production, vol. 249, 2020.

[41] J. Zhang and M. Cao, "Strategy competition dynamics of multi-agent systems in the framework of evolutionary game theory," IEEE Transactions on Circuits and Systems II: Express Briefs, vol. 67, no. 1, pp. 152-156, 2020.

[42] H. Cui, T. Zhao, and P. Tao, "Evolutionary game study on the development of green agriculture in China based on ambidexterity theory perspective," Polish Journal of Environmental Studies, vol. 28, no. 3, pp. 1093-1104, 2019.

[43] F. Gobet and G. Sala, "How artificial intelligence can help us understand human creativity," Frontiers in Psychology, vol. 10, Article ID 1401, 2019.

[44] X. Li, F. Wang, and X. Gu, "Understanding the roles of ICT enterprises in promoting the ICT industry ecosystem in education: case studies from China," British Journal of Educational Technology, vol. 50, no. 3, pp. 1151-1172, 2019.

[45] F. N. Zatta, E. T. Filho, F. C. De Campos, and R. R. Freitas, "Operational competencies and relational resources: a multiple case study," RAUSP Management Journal, vol. 54, no. 3, pp. 305-320, 2019. 